\title{
Performance Analysis on Fault Tolerant
}

\section{Control System}

\author{
Jong-Yeob Shin \\ National Institute of Aerospace, Hampton, VA 23666 \\ Christine Belcastro \\ NASA Langley Research Center, Hampton, VA 23681
}

\begin{abstract}
In a fault tolerant control (FTC) system, a parameter varying FTC law is reconfigured based on fault parameters estimated by fault detection and isolation (FDI) modules. FDI modules require some time to detect fault occurrences in aero-vehicle dynamics. In this paper, an FTC analysis framework is provided to calculate the upper bound of an induced- $\mathcal{L}_{2}$ norm of an FTC system with existence of false identification and detection time delay. The upper bound is written as a function of a fault detection time and exponential decay rates and has been used to determine which FTC law produces less performance degradation (tracking error) due to false identification. The analysis framework is applied for an FTC system of a HiMAT (Highly Maneuverable Aircraft Technology) vehicle.
\end{abstract}

Index Terms

fault tolerant control system, linear parameter varying system, HiMAT vehicle.

\section{INTRODUCTION}

In the past decades, there has been interest in a fault tolerant control (FTC) system which has the ability to detect actuator/sensor faults automatically and to prevent faults from developing into a total system failure. Especially in designing a flight control system, an active FTC system has been researched for achieving single aircraft accident prevention [1]-[4]. An active FTC system consists of an FTC law, a fault detection and isolation (FDI) module and a supervisory system. An FTC law should react to actuator/sensor faults through reconfi guration and an FDI

Staff Scientist, j.y.shin@larc.nasa.gov

Researcher, Dynamic Systems and Control Branch 
module should detect actuator/sensor fault occurrences. Based on the information provided by an FDI module, a supervisory system decides which actuator/sensor is faulty and sends a signal to an FTC law for reconfi guration.

In general, an active FTC law is designed, based on an open-loop system modeled as a function of fault parameters under the assumption that they are immediately identifi ed by an FDI module. Recently, using linear matrix inequality (LMI) optimization solutions [2]-[4], an active FTC law is synthesized in the form of a linear parameter varying (LPV) system whose dynamics vary as scheduling parameters change. Open-loop dynamics are modeled as an LPV system in which scheduling parameters are fault parameters that represent fault occurrences at actuators/sensors. An LPV-FTC law designed based on the open-loop system can robustly stabilize a closed-loop system and achieve desired performance during a fault occurrence under the assumption that fault parameters are measured in real-time.

Typically, there is always some level of time-delay to detect faults regardless of FDI algorithms such as an extended Kalman FDI fi lter [4] or an LPV-FDI fi lter in which a fault detection signal is calculated based on residual signal [5]. During a time-delay interval, an open-loop system is in a faulty condition but the information provided by an FDI module implies that the system is in a healthy condition. It is also possible that during a time interval an FDI module and a supervisory system may produce false identifi cation on healthy actuator/sensors, which may lead the system to be unstable at the moment.

Since an FDI module and an FTC law are individually designed, without considering the other dynamics [3], [4], it is required to analyze a whole FTC system including both an FTC law and an FDI module, before they are implemented into a real system. A typical way of analyzing an FTC system is full nonlinear simulation with the predefi ned command inputs (not all possible command inputs), for possible fault scenarios. After detailed simulations, an FTC system may be validated for possible fault scenarios with expensive computational costs. There should be an alternative analysis method to detailed simulations to provide a certain criteria related with characteristics of an FDI module such as time-delay and possible false identifi cation. In this paper, an FTC analysis framework is suggested to describe performance degradation in terms of induced $\mathcal{L}_{2}$ norm of a system due to possible false identifi cation and time delay in fault detection. Its upper bound is calculated by using LMI optimization and can be used to determine which FTC law has less worst-case performance degradation due to possible false identifi cation and possible time delay without detailed simulations. In this paper, it is demonstrated by applying the FTC analysis 
framework to a HiMAT FTC system designed in Ref. [4].

This paper contains the following sections. In section 2, an FTC system analysis problem is stated and an analysis methodology is described in section 3. In section 4, a HiMAT FTC system analysis is demonstrated. In section 5, this paper is concluded with a brief summary.

\section{Problem Statement}

To describe analysis problems on an FTC system, the general structure of an FTC system is briefly described here. As shown in Figure 1, an FTC system consists of an FTC law, an FDI module and a supervisory system (logics). When a fault occurs, the FDI module and supervisory system (logics) detect it and generate signals for evaluating/reconfi guring the FTC law. The FTC law is designed as an LPV system whose dynamics vary as scheduling parameters change [4], [6], [7]. The stability and performance level of a closed-loop system may change when a fault occurs, since the FDI model requires some time to detect fault. Hereafter, the required time is called detection time for an FDI module. During detection time, open-loop dynamics are in a faulty condition but the designed control law is not reconfigured yet for it. It implies that there exists a moment when the closed-loop system is not in the predicted closed-loop dynamics set used in the LPV control synthesis procedure.

In an FTC analysis framework, a closed-loop system is modeled as functions of fault parameters $\rho(t) \in \mathcal{R}^{n_{s}}$ and estimated fault parameters $\bar{\rho}(t) \in \mathcal{R}^{n_{s}}$ by an FDI module and a supervisory system since open-loop dynamics $P(\rho)$ are dependent on actual fault parameters and the control law $K(\bar{\rho})$ must use the estimates. The closed-loop system can be written as:

$$
\begin{aligned}
\dot{x} & =A(\rho, \bar{\rho}) x+B(\rho, \bar{\rho}) d, \\
e & =C(\rho, \bar{\rho}) x+D(\rho, \bar{\rho}) d,
\end{aligned}
$$

where states $x \in \mathcal{R}^{n_{x}}$, disturbances $d \in \mathcal{R}^{n_{d}}$ and errors $e \in \mathcal{R}^{n_{e}}$. Note that fault parameters and estimated fault parameters are treated as parameters independent of each other in a closed-loop system to capture the dynamic variations due to possible false identifi cation. Using a robust LPV control synthesis methodology in Refs. [4], [8] a control law $K(\bar{\rho})$ is designed to robustly stabilize open-loop dynamic variations $P\left(\rho \pm \delta_{\rho}\right)$ with $\rho$ satisfying the 
condition:

$$
\rho(t) \in \mathcal{S}\left(\bar{\rho}, \delta_{\rho}\right):=\left\{\rho(t) \mid\|\rho(t)-\bar{\rho}(t)\| \leq \delta_{\rho}, 0 \leq \delta_{\rho}\right\}
$$

where $\delta_{\rho}$ is an estimation error bound. Note that the condition is not valid during the detection time.

The entire parameter space of a closed-loop system is defi ned as

$$
\mathcal{P}:=\left\{(\rho(t), \bar{\rho}(t)) \mid \rho(t) \in \mathcal{F}_{\rho}, \bar{\rho}(t) \in \mathcal{F}_{\bar{\rho}}\right\}
$$

where $\mathcal{F}_{\rho}$ and $\mathcal{F}_{\bar{\rho}}$ are bounded compact sets in $R^{n_{s}}$ and can be divided into subspaces $\mathcal{P}_{i}$ such that

$$
\begin{aligned}
& \mathcal{P}=\bigcup_{i=1}^{m} \mathcal{P}_{i}:=\mathcal{P}_{1} \cup \mathcal{P}_{2} \cdots \cup \mathcal{P}_{m}, \\
& \emptyset \quad=\mathcal{P}_{i} \cap \mathcal{P}_{j}, \quad i \neq j, \quad i \in \mathcal{I}, \quad j \in \mathcal{I}, \quad \mathcal{I}:=\{1,2, \cdots, m\} .
\end{aligned}
$$

For example, let's defi ne a subspace $\mathcal{P}_{1}$ as $\mathcal{P}_{1}:=\left\{(\rho, \bar{\rho}) \mid \rho(t) \in \mathcal{S}\left(\bar{\rho}, \delta_{\rho}\right)\right\}$. When parameter trajectories $(\rho(t), \bar{\rho}(t))$ stay in $\mathcal{P}_{1}$, it implies that the closed-loop system should be stable because the controller is designed for that. During detection time, the condition (2) is not satisfi ed generally. In that case, parameter subspaces $\mathcal{P}_{2} \cdots \mathcal{P}_{m}$ can be defi ned by a reader based on dynamic changes in the closed-loop system. Without loss of generality, a subspace $\mathcal{P}_{m}$ is defi ned as the set of parameter trajectories $(\rho(t), \bar{\rho}(t))$ when the closed-loop system is locally unstable along the trajectories.

\section{Definition 1 Local stability}

Suppose all matrices $A(\rho, \bar{\rho})$ at fixed $\rho$ and $\bar{\rho}$ in a subspace $\mathcal{P}_{i}$ are stable. Then the system is called locally stable in the subspace $\mathcal{P}_{i}$.

To represent dynamic variations of the system in Eq (1) over each parameter subspace, a duration time over each subspace is defi ned as follows [9]:

Definition 2 Duration time $T_{p_{i}}$ over each parameter subspace :

$$
T_{p_{i}}\left(t_{o}, t\right)=T_{o_{i}}+\int_{t_{o}}^{t} \sigma_{i}(\rho(s), \bar{\rho}(s)) d s, \quad \forall t>t_{o}>0
$$




$$
\sigma_{i}(\rho(s), \bar{\rho}(s))= \begin{cases}0, & (\rho(s), \bar{\rho}(s)) \notin \mathcal{P}_{i}, \\ 1, & (\rho(s), \bar{\rho}(s)) \in \mathcal{P}_{i}, \quad i \in \mathcal{I} .\end{cases}
$$

A duration time is bounded as

$$
T_{o_{i}} \leq T_{p_{i}} \leq T_{o_{i}}+\alpha_{i}\left(t-t_{o}\right)
$$

where $0 \leq \alpha_{i} \leq 1$ and $0 \leq T_{o_{i}}$. The constant $\alpha_{i}$ represents a ratio of the duration time in the $i$-th subspace to the total time and $T_{o_{i}}$ is the duration time for a system to stay in the $i$-th subspace during the interval $\left[0, t_{o}\right]$. Note that $\sum_{i=1}^{m} \alpha_{i}=1$ and $\sum_{i=1}^{m} T_{o_{i}}=t_{o}$.

Consider the case that the system is assumed to be locally unstable in the $m$-th subspace. The constant $\alpha_{m}<1$ plays an important role in stability analysis used to fi nd an asymptotic stability ratio [9], which is related to stability margin of the system.

\section{ANALysis Method}

\section{A. Stability analysis}

Suppose a system in Eq. (1) is locally stable in the set of $\bigcup_{i=1}^{m-1} \mathcal{P}_{i}$ and locally unstable in the parameter subspace $\mathcal{P}_{m}$

Proposition 1 [9]: Suppose there exists a positive definite matrix $P(\bar{\rho})$ such that

$$
\begin{aligned}
& A^{T}(\rho, \bar{\rho}) P(\bar{\rho})+P(\bar{\rho}) A(\rho, \bar{\rho})+\dot{P}(\bar{\rho}) \leq-\lambda_{i} P(\bar{\rho}), \quad(\rho, \bar{\rho}) \in \mathcal{P}_{i}, \text { locally stable } \\
& A^{T}(\rho, \bar{\rho}) P(\bar{\rho})+P(\bar{\rho}) A(\rho, \bar{\rho})+\dot{P}(\bar{\rho}) \leq \kappa P(\bar{\rho}), \quad(\rho, \bar{\rho}) \in \mathcal{P}_{m}, \text { locally unstable }
\end{aligned}
$$

where

$$
0 \leq \kappa, \quad 0 \leq \lambda_{i} \leq \lambda_{1}, \quad i \in \mathcal{I}-\{m\}
$$

The system in Eq. (1) is exponentially stable with a decay rate:

$$
\lambda=\lambda_{1}-\sum_{i=2}^{m-1}\left(\lambda_{1}-\lambda_{i}\right) \alpha_{i}-\left(\lambda_{1}+\kappa\right) \alpha_{m}
$$


under the condition:

$$
\alpha_{m}<\alpha_{t}:=\frac{\lambda_{\min }}{\lambda_{\min }+\kappa}
$$

where $\lambda_{\min }=\min \left\{\lambda_{i}\right\}, \quad i \in \mathcal{I}-\{m\}$.

Proof: Set a Lyapunov function as $V=x^{T}(t) P(\bar{\rho}) x(t), P(\bar{\rho}) \in \mathcal{R}^{n_{x} \times n_{x}}$. Using Eq. (8), the time derivative of the Lyapunov function is

$$
\dot{V} \leq \begin{cases}-\lambda_{i} V, & (\rho, \bar{\rho}) \in \mathcal{P}_{i}, \quad i \in \mathcal{I}-\{m\} \\ \kappa V, & (\rho, \bar{\rho}) \in \mathcal{P}_{m} .\end{cases}
$$

From Eq. (12),

$$
V \leq e^{-\lambda_{1}\left(t-t_{o}-\sum_{i=2}^{m} T_{p_{i}}\right)-\sum_{i=2}^{m-1} \lambda_{i} T_{p_{i}}+\kappa T_{p_{m}}} V\left(t_{o}\right), \quad \forall t \geq t_{o} \geq 0 .
$$

Taking the upper bound of the duration time over each parameter subspace, Eq. (13) is rewritten as:

$$
V \leq V\left(t_{o}\right) e^{-\lambda\left(t-t_{o}\right)+\sum_{i=2}^{m-1}\left(\lambda_{1}-\lambda_{i}\right) T_{o_{i}}+\left(\lambda_{1}+\kappa\right) T_{o_{m}}}
$$

where

$$
\lambda:=\lambda_{1}-\sum_{i=2}^{m-1}\left(\lambda_{1}-\lambda_{i}\right) \alpha_{i}-\left(\lambda_{1}+\kappa\right) \alpha_{m} .
$$

Without loss of generality, the constant $\lambda_{1}$ can be set as $\max \left\{\lambda_{i}\right\}, i \in \mathcal{I}-\{m\}$. Thus, the term $\lambda_{1}-\lambda_{i}$ is always positive over the parameter subspaces $\mathcal{P}_{i}, i \in \mathcal{I}-\{1, m\}$. The lower bound of the constant $\lambda$ is

$$
\lambda \geq \lambda_{t}:=\lambda_{1}-\sum_{i=2}^{m-1}\left(\lambda_{1}-\lambda_{\min }\right) \alpha_{i}-\left(\lambda_{1}+\kappa\right) \alpha_{m} .
$$

When the condition in Eq. (11) is satisfied, it can be easily shown that the system is exponentially stable.

In the FTC system analysis, the constant $\alpha_{t}$ can be interpreted as tolerance of instability during a detection time interval. For example, $\alpha_{t}=0.1$ implies that the closed-loop system can stay in the parameter subspace $\mathcal{P}_{m}$ for at most $10 \%$ of the total interest time without loss of exponential stability. Given fault tolerant control laws, we can analyze stability of the closed-loop system in terms of a constant $\alpha_{t}$ value. The stability analysis problem can be 
formulated into an optimization problem as:

$$
\max _{\kappa>0, \lambda_{\min }>0} \alpha_{t}, \quad \text { s.t. } \quad \text { Eq.(8). }
$$

The optimization is solved by checking feasibility of the LMI constraints of Eq. (8) using the LMI Toolbox [11] and line searching over $\lambda_{i}$ and $\kappa$ values.

\section{B. Performance analysis}

For a closed-loop system of Eq. (1), an induced- $\mathcal{L}_{2}$ norm is defi ned as:

$$
\sup _{(\rho, \bar{\rho}) \in \mathcal{P}, d \in \mathcal{L}_{2},\|d\|_{2} \neq 0} \frac{\|e\|_{2}}{\|d\|_{2}}
$$

In this paper, the performance level of an FTC system with false identifi cation and detection time delay is calculated in terms of the induced- $\mathcal{L}_{2}$ norm as follows:

Proposition 2: Suppose there exists a positive definite matrix $P(\bar{\rho}) \in \mathcal{R}^{n_{x} \times n_{x}}$ such that

$$
\begin{aligned}
& {\left[\begin{array}{ccc}
A^{T} P+P A+\dot{P}+\lambda_{i} P & P B & \gamma^{-1} C^{T} \\
B^{T} P & -I & \gamma^{-1} D^{T} \\
\gamma^{-1} C & \gamma^{-1} D & -I
\end{array}\right]<0, \quad(\rho, \bar{\rho}) \in \mathcal{P}_{i}, \quad i \in \mathcal{I}-\{m\},} \\
& {\left[\begin{array}{ccc}
A^{T} P+P A+\dot{P}-\kappa P & P B & \gamma^{-1} C^{T} \\
B^{T} P & -I & \gamma^{-1} D^{T} \\
\gamma^{-1} C & \gamma^{-1} D & -I
\end{array}\right]<0, \quad(\rho, \bar{\rho}) \in \mathcal{P}_{m} .}
\end{aligned}
$$

The induced- $\mathcal{L}_{2}$ norm from $d$ to e of the closed-loop system is no larger than $M_{\gamma}$ where

$$
\begin{aligned}
& M_{\gamma}=\gamma \sqrt{\frac{e^{\sum_{i=2}^{m-1}\left(\lambda_{1}-\lambda_{i}\right) T_{o_{i}}+\left(\lambda_{1}+\kappa\right) T_{o_{m}} \lambda_{1}}}{\lambda}}, \\
& \lambda=\lambda_{1}-\sum_{i=2}^{m-1}\left(\lambda_{1}-\lambda_{i}\right) \alpha_{i}-\left(\lambda_{1}+\kappa\right) \alpha_{m} .
\end{aligned}
$$

Under the condition $\alpha_{m}<\alpha_{t}:=\frac{\lambda_{\min }}{\left(\lambda_{\min }+\kappa\right)}, \lambda>0$. 
Proof: Set $V=x^{T}(t) P(\bar{\rho}) x(t)$. Then using Eq. (19), the time derivative of the Lyapunov function $V$ is

$$
\dot{V} \leq \begin{cases}-\lambda_{i} V+\|d\|^{2}-\gamma^{-2}\|e\|^{2}, & (\rho, \bar{\rho}) \in \mathcal{P}_{i}, \\ \kappa V+\|d\|^{2}-\gamma^{-2}\|e\|^{2}, & (\rho, \bar{\rho}) \in \mathcal{P}_{m} .\end{cases}
$$

Using Eq. (22), $V$ is rewritten as:

$$
V(t) \leq e^{f\left(t_{0}, t\right)} V\left(t_{o}\right)+\int_{t_{o}}^{t} e^{f(s, t)}\left(\|d(s)\|^{2}-\gamma^{-2}\|e(s)\|^{2}\right) d s
$$

where

$$
f(s, t)=-\lambda_{1}\left(t-s-\sum_{i=2}^{m} T_{p_{i}}(s, t)\right)-\sum_{i=2}^{m-1} \lambda_{i} T_{p_{i}}(s, t)+\kappa T_{p_{m}}(s, t)
$$

Since $V(t)>0, \quad \forall t \geq t_{o} \geq 0$, the following inequality is extracted from Eq. (23):

$$
\gamma^{-2} \int_{t_{o}}^{t} e^{\lambda_{1}(t-s)}\|e(s)\|^{2} d s \leq e^{f\left(t_{o}, t\right)} V\left(t_{o}\right)+\int_{t_{o}}^{t} e^{f(s, t)}\|d(s)\|^{2} d s, \quad \forall t \geq t_{o} \geq 0
$$

Using Eq. (7) and the defi nition of $\lambda$ in Eq. (21), it is derived that

$$
\begin{aligned}
\gamma^{-2} \int_{t_{o}}^{t} e^{-\lambda_{1}(t-s)}\|e(s)\|^{2} d s \leq & V\left(t_{o}\right) e^{\sum_{i=2}^{m-1}\left(\lambda_{1}-\lambda_{i}\right) T_{o_{i}}+\left(\lambda_{1}+\kappa\right) T_{o m}-\lambda\left(t-t_{o}\right)} \\
& +\int_{t_{o}}^{t} e^{\sum_{i=2}^{m-1}\left(\lambda_{1}-\lambda_{i}\right) T_{o_{i}}+\left(\lambda_{1}+\kappa\right) T_{o_{m}}-\lambda(t-s)}\|d(s)\|^{2} d s .
\end{aligned}
$$

Integrating both sides of Eq. (25) over the interval $\left[t_{o}, \infty\right)$ leads to

$$
\frac{\gamma^{-2}}{\lambda_{1}} \int_{t_{o}}^{\infty}\|e(s)\|^{2} d s \leq \frac{V\left(t_{o}\right)}{\lambda} e^{\sum_{i=2}^{m-1}\left(\lambda_{1}-\lambda_{i}\right) T_{o_{i}}+\left(\lambda_{1}+\kappa\right) T_{o m}}+\frac{1}{\lambda} e^{\sum_{i=2}^{m-1}\left(\lambda_{1}-\lambda_{i}\right) T_{o_{i}}+\left(\lambda_{o}+\kappa\right) T_{o m}} \int_{t_{o}}^{\infty}\|d(s)\|^{2} d s
$$

Thus, the upper bound $M_{\gamma}$ of the induced- $\mathcal{L}_{2}$ norm is

$$
M_{\gamma}=\gamma \sqrt{\frac{e^{\sum_{i=2}^{m-1}\left(\lambda_{1}-\lambda_{i}\right) T_{o_{i}}+\left(\lambda_{1}+\kappa\right) T_{o_{m}}} \lambda_{1}}{\lambda}} .
$$


The constant $M_{\gamma}$ represents the upper bound of the worst-case performance level due to false identifi cation and the time delay. Thus, the performance analysis problem is formulated into an optimization problem:

$$
\min _{\lambda_{i}>0, \kappa \geq 0, \gamma>0, P>0} M_{\gamma} \text {, s.t. Eq.(19). }
$$

The optimization problem Eq.(28) is solved by line searching over pre-defi ned $\lambda_{i}$ and $\kappa$ ranges and using the LMI Toolbox [11]. First, feasible ranges of $\lambda_{i}$ and $\kappa$ are chosen based on few test run results. Second, the minimum value of $\gamma$ is calculated using the LMI Toolbox [11] at fi xed $\lambda_{i}$ and $\kappa$ values with the constraint Eq. (19). Third, an upper bound $M_{\gamma}$ is calculated with given time delay values $T_{o_{i}}$ and steps 2 and 3 are repeated to fi nd the minimum value of $M_{\gamma}$ over the pre-defi ned $\lambda_{i}$ and $\kappa$ ranges.

\section{EXAMPLE}

\section{A. HiMAT FTC System}

The HiMAT FTC system taken from Ref. [4] is briefly described here before applying the analysis framework suggested in section 3. The model has two inputs $u$ : elevons $\delta_{e}$ and canards $\delta_{c}$; two outputs $y$ : angle of attack $\alpha$ in radians and pitch angle $\theta$ in radians; and four states $x$ : velocity $V$ in $\mathrm{ft} / \mathrm{sec}$, angle of attack $\alpha$, pitch rate $q$ in $\mathrm{rad} / \mathrm{sec}$, and pitch angle $\theta$. The LPV model of the HiMAT vehicle [4] is

$$
\begin{gathered}
\dot{x}=A x+B\left[\begin{array}{ll}
\tau_{1} & 0 \\
0 & \tau_{2}
\end{array}\right] u+B\left[\begin{array}{ll}
0.1 & 0 \\
0 & 0.1
\end{array}\right] w, \quad z=u, \quad y=C x \\
w=\Delta z, \quad \Delta=\left[\begin{array}{ll}
\delta_{1} & 0 \\
0 & \delta_{2}
\end{array}\right],\left|\delta_{1}\right| \leq 1,\left|\delta_{2}\right| \leq 1
\end{gathered}
$$

where the detailed elements of the system matrices $A, B$, and $C$ are in Ref. [4]. It is noticed from Eq. (29) that faults on the actuators are modeled as control sensitivity variations (a function of fault parameters $\tau_{1}$ and $\tau_{2}$ ) under the assumption that estimation error bound is 0.1 . Consider a failure case that one actuator is failed at a time to 
keep controllability of the open-loop system non-zero. To represent one fault at a time, the scheduling parameter of the LPV model is chosen as $\rho$ such that

$$
\begin{aligned}
0 \leq \rho<1 \quad: \quad 0 \leq \tau_{1}<1, \quad \tau_{2}=1 \quad \text { Elevon failure } \\
\rho=1 \quad: \quad \tau_{1}=1, \quad \tau_{2}=1 \\
1<\rho \leq 2 \quad: \quad \tau_{1}=1, \quad 0 \leq \tau_{2}<1 \quad \text { Canard failure. }
\end{aligned}
$$

For the detailed fault models, the reader is referred to Ref. [4].

The on-line FDI module and FTC control laws are taken from Ref. [4] and are very briefly described here. The fault parameters $\tau_{1}$ and $\tau_{2}$ are estimated by the on-line FDI module designed using a two-stage extended Kalman fi lter [4], [12]. Using the same logics in Eq. (31), the estimated scheduling parameter $\bar{\rho}$ is calculated from estimated fault parameters $\bar{\tau}_{1}$ and $\bar{\tau}_{2}$. The LPV-FTC laws $K_{A}$ and $K_{B}$ are designed using the conventional LPV control synthesis [7] and the robust LPV control synthesis [4], respectively. In the conventional LPV synthesis method, it is assumed that scheduling parameters are exactly measured. In the robust LPV synthesis method [4] scheduling parameters can be estimated within a given estimation error bound. In the control synthesis process, the design objective is to reduce pitch angle command tracking error for both controllers. Note that false identifi cation and estimate time-delay are not considered in the control synthesis procedure.

In this example, it will be determined which controller can generate less pitch angle command tracking error due to false identifi cation without detailed simulations. In this model, false identifi cation cases can be 1) canard failure indicated by the FDI module for actual elevator failure or 2) elevator failure indicated for actual canard failure for a short time interval. Here only the first case of false identifi cation is be analyzed because it can severely affect the closed-loop dynamics. Note that the closed-loop system is locally unstable for the fi rst case but not for the second case.

To analyze the false identifi cation $(\rho=2$ and $\bar{\rho} \approx 0)$, the parameter set $\mathcal{P}$ is divided into three subspaces such 
as

$$
\begin{aligned}
& \mathcal{P}_{1}=\{(\rho, \bar{\rho}) \mid \rho=2, \quad 0.9<\bar{\rho} \leq 2\} \\
& \mathcal{P}_{2}=\{(\rho, \bar{\rho}) \mid \rho=2, \quad 0.1<\bar{\rho} \leq 0.9\} \\
& \mathcal{P}_{3}=\{(\rho, \bar{\rho}) \mid \rho=2, \quad 0.0 \leq \bar{\rho} \leq 0.1\} .
\end{aligned}
$$

The parameter subspaces are determined based on the dynamic variations of the closed-loop system. More specifically, the subspace $\mathcal{P}_{1}$ is set because the LPV control law is designed for the predicted closed-loop dynamics in the subspace $\mathcal{P}_{1}$ [4]. The subspace $\mathcal{P}_{2}$ is set because the closed-loop system is locally stable but the closed-loop dynamics set in the subspace $\mathcal{P}_{2}$ is not considered in the control synthesis procedure. The subspace $\mathcal{P}_{3}$ is set for the false identifi cation case in which the closed-loop system is locally unstable in $\mathcal{P}_{3}$.

\section{B. Stability analysis on the HiMAT FTC system}

In this subsection, stability of the HiMAT FTC system is analyzed for the false identifi cation that leads to the closed-loop system being locally unstable. The LMI constraints in Eq. (8) are evaluated at grid points $\bar{\rho} \in$ $\{0,0.1,0.2, \cdots, 2\}$ over the parameter subspaces defi ned in Eq. (32). To solve the optimization problem in Eq. (17), the ranges of $\lambda_{1}, \lambda_{2}$, and $\kappa$ are defi ned as $0.01 \leq \lambda_{1} \leq 0.1,0.01 \leq \lambda_{2} \leq 0.1$, and $5 \leq \kappa \leq 10$, respectively. With fi xed $\lambda_{1}, \lambda_{2}$, and $\kappa$ values in each range, the feasibility of the LMI constraints is checked with a constant matrix $P$ and a parameter dependent matrix $P(\bar{\rho})$ for reducing conservatism in LMI solution [7], respectively. To use a

parameter dependent matrix $P(\bar{\rho})$, the time derivative $\dot{\bar{\rho}}$ is required to determine $\dot{P}=\dot{\bar{\rho}} \frac{\partial P(\bar{\rho})}{\partial \bar{\rho}}$. In this example, the basis functions for $\mathcal{P}(\bar{\rho})$ and the bound of time derivative $\dot{\bar{\rho}}$ are taken from Ref. [4]. Note that it is still unknown how to choose optimal basis functions in LPV control synthesis.

The calculated $\alpha_{t}$ values are $5 \times 10^{-3}$ for the control $K_{A}$ and $8 \times 10^{-3}$ for the control $K_{B}$ using $\mathcal{P}$ or $\mathcal{P}(\bar{\rho})$. There is not much difference using between constant $\mathcal{P}$ and $\mathcal{P}(\bar{\rho})$ in stability analysis results. It is noticed from the analysis results that the closed-loop system can be stable if the false identifi cation occurs every $100 \mathrm{sec}$ time with duration $0.5 \mathrm{sec}$. Both controllers have similar effects on closed-loop stability due to the false identifi cation. 
TABLE I

PERFormance ANALYSis RESUlts

\begin{tabular}{c|cccc}
\hline \hline \multicolumn{5}{c}{ constant matrix $P$} \\
\hline Controller & $\lambda_{1}$ & $\lambda_{2}$ & $\kappa$ & $\gamma$ \\
\hline$K_{A}$ & $3 \times 10^{-3}$ & $1 \times 10^{-3}$ & 9.4 & 0.42 \\
$K_{B}$ & $6 \times 10^{-4}$ & $1 \times 10^{-4}$ & 5.85 & 2.14 \\
\hline \multicolumn{5}{c}{ parameter dependent $P(\bar{\rho})$} \\
\hline Controller & $\lambda_{1}$ & $\lambda_{2}$ & $\kappa$ & $\gamma$ \\
\hline$K_{A}$ & $6 \times 10^{-4}$ & $1 \times 10^{-4}$ & 9.4 & 0.34 \\
$K_{B}$ & $6 \times 10^{-4}$ & $1 \times 10^{-4}$ & 3.7 & 0.63 \\
\hline \hline
\end{tabular}

\section{Performance analysis on the HiMAT FTC system}

In this section, the upper bound of the induced- $\mathcal{L}_{2}$ norm from pitch angle command to tracking error of the FTC system is calculated due to the false identification. Using line searching over the $\lambda_{1}, \lambda_{2}$, and $\kappa$ ranges, the analysis results are shown in Table I. Using the analysis results in Table I for each controller, the $M_{\gamma}$ variations are calculated due to false identifi cation time $T_{o_{3}}$ variations using Eq. (27) and are shown in Figure 2. Note that the duration time $T_{o_{2}}$ is set as $0.2 \mathrm{sec}$ here. Since $\lambda_{1}$ and $\lambda_{2}$ are similar values, the time $T_{o_{2}}$ does not affect much the upper bound $M_{\gamma}$. It is observed from Figure 2 that performance analysis results are signifi cantly different using a constant matrix $P$ and a parameter dependent matrix $P(\bar{\rho})$. Recall that using a parameter dependent matrix $P(\bar{\rho})$ can reduce conservatism in results of LMI [7]. It is easily noticed from Figure 2 that when a false identifi cation time is short $\left(T_{o_{3}}<0.22 \mathrm{sec}\right)$, the $M_{\gamma}$ with the $K_{A}$ control law is less than that with the $K_{B}$ control law. When the time is long $\left(T_{o_{3}}>0.22 \mathrm{sec}\right)$, the $M_{\gamma}$ values are vice versa. The analysis results imply that the $K_{A}$ control law leads to less tracking error when $T_{o_{3}}<0.22 \mathrm{sec}$ and the $K_{B}$ control law leads to less tracking error when $T_{o_{3}}>0.22 \mathrm{sec} . \quad$ To validate the FTC system performance analysis results, the closed-loop system with a step pitch angle command at $1 \mathrm{sec}$ is simulated with assumption of false identifi cation detection time $T_{0_{3}}=0.1,0.2,0.4$ and $0.8 \mathrm{sec}$ for each control law. In each simulation, canards are failed at $1 \mathrm{sec}$. The tracking error time histories are plotted in Figure 3 for each case. Obviously noticeable is that the error norm of $\left\|\theta-\theta_{c m d}\right\|_{2}$ with the $K_{A}$ 
control law is larger than that with the $K_{B}$ control law at the $T_{o_{3}}=0.4$ and $0.8 \mathrm{sec}$ cases. Also, the error norm with the $K_{B}$ control law is larger than that with the $K_{A}$ control law at the $T_{o_{3}}=0.1$ and $0.2 \mathrm{sec}$ cases. It shows that the parameter dependent analysis results in Figure 2 correspond to the simulation results in Figure 3.

\section{CONCLUSION}

In this paper, the FTC system analysis problem is formulated into an optimization problem with LMI constraints which are evaluated at grid points over the stable/unstable parameter subspaces. From the stability analysis, the stability margin of an FTC system can be calculated for possible false identifi cation when a fault occurs. From the performance analysis, the upper bound of the induced- $\mathcal{L}_{2}$ norm of the FTC system represents worst-case performance during the detection time interval of FDI modules. The upper bound is calculated as a function of the detection time interval and exponential decay rates over each parameter subspace. It indicates performance degradation due to false identifi cation. The usage of the FTC system analysis is demonstrated via analysis of the FTC HiMAT system.

\section{ACKNOWLEDGMENTS}

This research was supported by National Aeronautics and Space Administration under NASA Contract No. NAS1-02117.

\section{REFERENCES}

[1] C. Belcastro and C. Belcastro, "Application of failure detection, identifi cation, and accommodation methods for improved aircraft safety," in Proceedings of American Control Conference. American Automatic Control Council, Evanston, IL, 2001, pp. 2623-2624.

[2] J. Chen, R.J. Patton, and Z. Chen, “An LMI approach to Fault-Tolerant Control of Uncertain System," in Proceeding of the 1998 International Symposium on Intelligent Control. Institute of Electrical and Electronics Engineers, Piscataway, N.J., 1998, pp. 175-180.

[3] S. Ganguli, A. Marcos, and G. Balas, "Reconfi gurable LPV Control Design for Boeing 747-100/200 Longitudinal Axis," in Proceedings of American Control Conference. Anchorage, AK: American Control Conference Council, Evanston, IL, 2002, pp. 3612-3617.

[4] J.-Y. Shin, N.E. Wu, and C. Belcastro, “Adaptive Linear Parameter Varying Control Synthesis for Actuator Failure,” Journal of Guidance, Control, and Dynamics, Vol. 27, No. 5, pp.787-794, 2004.

[5] I. Szaszi, A. Marcos, G. Balas, and J. Bokor, "LPV Detection Filter Design for Boeing 747-100/200," in AIAA Guidance, Navigation and Control, no. AIAA 2002-4957. American Institute of Aeronautics and Astronautics, 2002. 
[6] J.-Y. Shin, "Worst-Case Analysis and Linear Parameter-Varying Gain-Scheduled Control of Aerospace Systems," Ph.D. dissertation, Department of Aerospace Engineering and Mechanics, University of Minnesota, 2000.

[7] F. Wu, "Control of Linear Parameter Varying Systems," Ph.D. dissertation, Department of Mechanical Engineering, University of California, Berkeley, 1995.

[8] P. Apkarian and R. Adams, "Advanced Gain-Scheduling Techniques for Uncertain Systems," IEEE Transactions on Control Systems Technology, vol. 6, no. 1, pp. 21-32, 1998.

[9] J. Hespanha, O. Yakimenko, I. Kaminer, and A. Pascoal, "LPV Systems with Brief Instabilities:Application to Integrated Vision/IMU Navigation," in IEEE Proceeding of the Conference on Decision and Control, 2001.

[10] J.-Y. Shin and C. Belcastro, "Analysis of A Fault Tolerant Control System: False Fault Detection Case," in 5th IFAC Symposium on Fault Detection, Supervision and Safety of Technical Processes, June 2003.

[11] P. Gahinet, A. Nemirovski, A.J. Laub, and M. Chilai, LMI Control Toolbox User's Guide. Natick, Mass: The MATH Works, 1995.

[12] J.K. Keller and M. Darouach, "Optimal two-stage Kalman fi lter in the presence of random bias," Automatica, vol. 33, no. 9, pp. 1745-1748, 1997. 


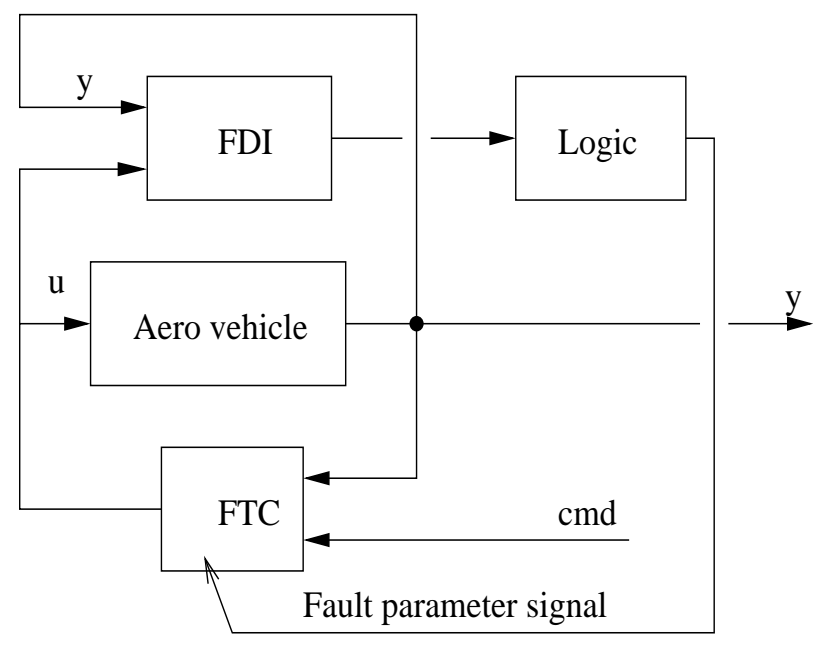

Fig. 1. Structure of a fault tolerant control system. 

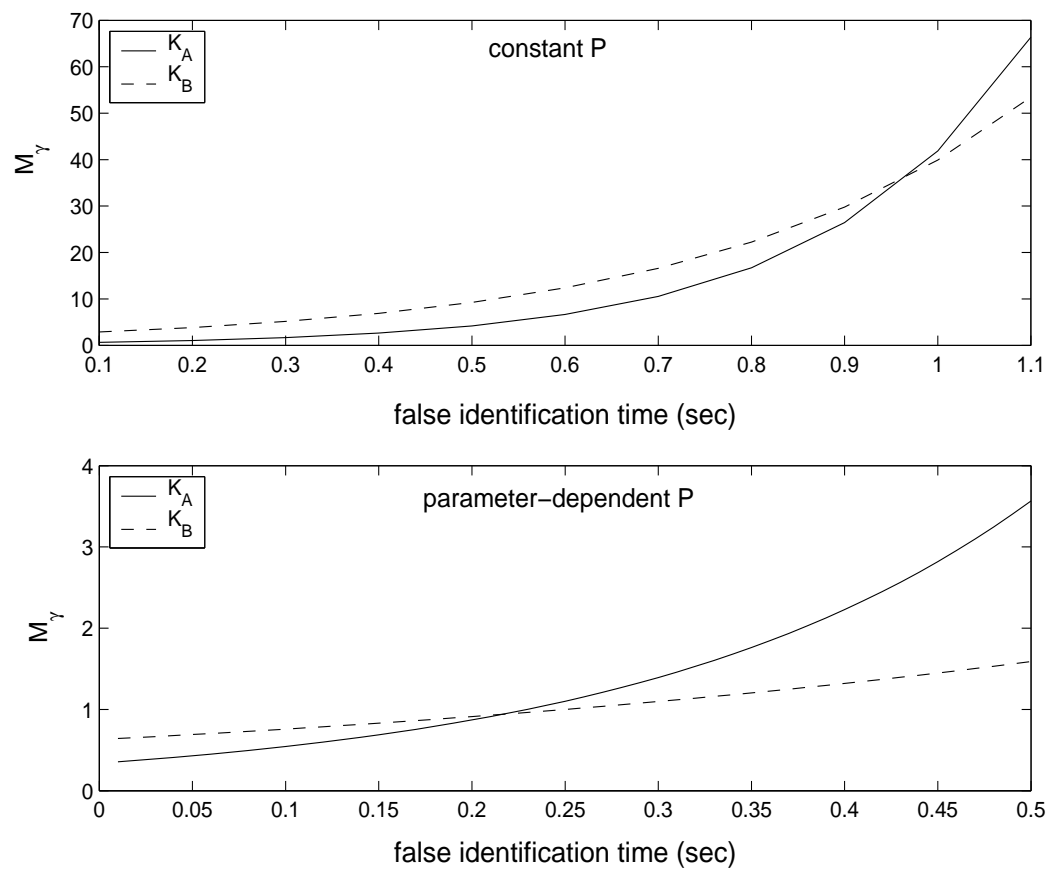

Fig. 2. Variations of $M_{\gamma}$ due to false identifi cation time $\left(T_{O_{3}}\right)$ changes.
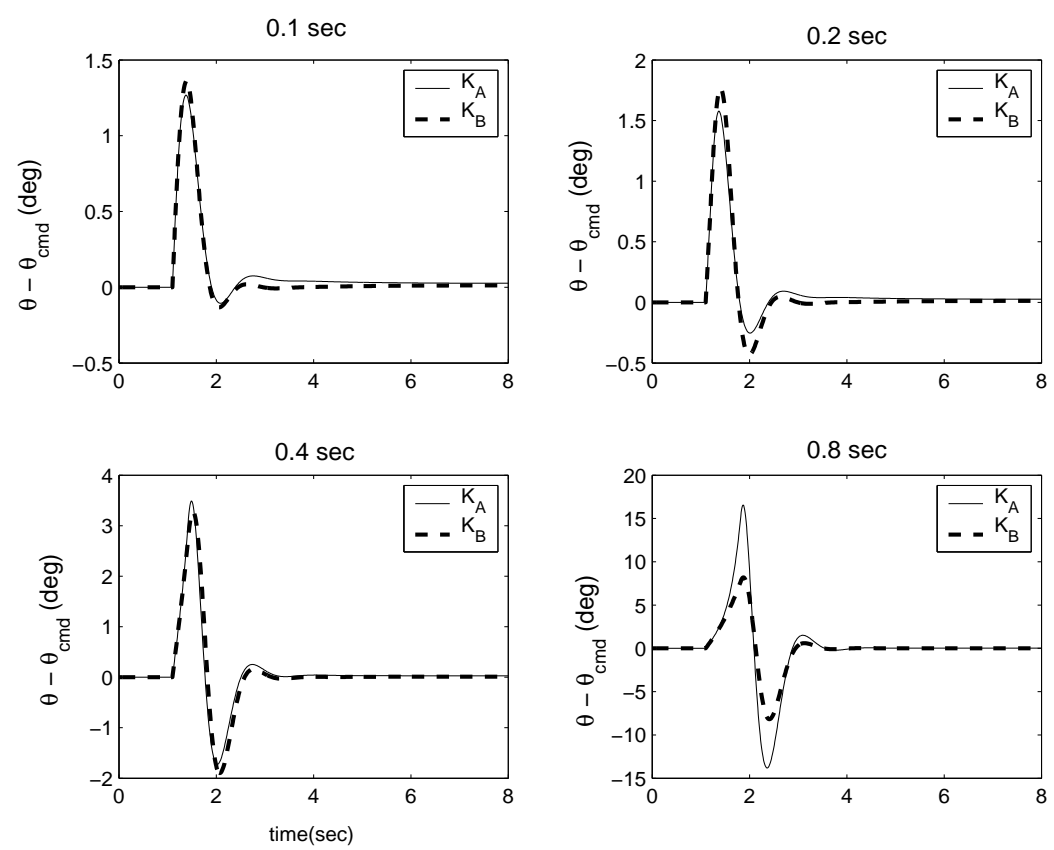

Fig. 3. Simulation results with different false identifi cation time values: $0.1,0.2,0.4$ and 0.8 sec. 